\title{
PROPIEDADES PSICOMÉTRICAS DE LA \\ ESCALA DE PROCRASTINACIÓN ACADÉMICA (EPA) \\ EN ESTUDIANTES DE PSICOLOGÍA DE AMBATO, ECUADOR
}

PSYCHOMETRIC PROPERTIES OF ACADEMIC PROCRASTINATION SCALE (EPA)

IN A PSYCHOLOGY'S STUDENTS GROUP FROM AMBATO, ECUADOR

Recibido: 12 de diciembre de 2017 | Aceptado: 24 de julio de 2018

DOI: 10.22199/507187475.2018.0003.00003

\section{RODRIGO MORETA-HERRERA1, TERESA DURÁN-RODRÍGUEZ2}

1., 2. PONTIFICIA UNIVERSIDAD CATÓLICA DEL ECUADOR, Ambato, Ecuador

\begin{abstract}
RESUMEN
OBJETIVO: Conocer las propiedades psicométricas de la Escala de Procrastinación Académica (EPA) en una muestra de estudiantes universitarios de la ciudad de Ambato, Ecuador. MÉTODO: Investigación de carácter instrumental, que analiza la estructura factorial, la fiabilidad y la validez temporal y de convergencia con la versión castellana de la Escala de Procrastinación Académica de Busko. MUESTRA: 290 estudiantes de la Pontificia Universidad Católica del Ecuador - Ambato (76,6\% mujeres). RESULTADOS: El Análisis Factorial muestra la estructura de la prueba con dos factores que explica el 53,1\% de la varianza con valores de B-S $X^{2}(53)=119,1 ; p<, 001 ; C F I=0,90 ; G F I=0,92 ; A G F I=0,89$ y RMSEA $=0,076 ; \mathrm{IC}: 90 \%[0,006-0,009]$. Además, es fiable entre las puntuaciones de los ítems $(\alpha=, 83)$ y estable en términos temporales $(r=, 778)$ con tres semanas de intervalo y converge de manera moderada con la Escala de Procrastinación de Tuckman $(r=$,594). CONCLUSIONES: La prueba de procrastinación académica muestra resultados favorables de validez y fiabilidad en estudiantes ecuatorianos.
\end{abstract}

PALABRAS CLAVE: Análisis factorial, fiabilidad, Procrastinación académica, validez.

\section{ABSTRACT}

AIM: Knowing the psychometric properties of Academic Procrastination Scale (EPA) in a sample of students of Ambato, Ecuador. METHOD: Instrumental research, which analyzes the factorial structure, reliability and temporal validity and convergence with the spanish version of the Busko Academic Procrastination Scale. SAMPLE: 290 university students of the Pontifical Catholic University of Ecuador - Ambato (76,6\% women). RESULTS: Factorial Analysis shows the test's structure with two factors explaining the $48,2 \%$ with values of $\mathrm{B}_{-} \mathrm{S} \mathrm{X}_{(53)}=119,1 ; \mathrm{p}<, 001$; $\mathrm{CFI}=0,90 ; \mathrm{GFI}=0,92 ; \mathrm{AGFI}=0,89$ y RMSEA $=0,076$; IC: $90 \%[0,006-$ $0,009]$. Moreover, is reliable between item scores $(\alpha=, 83)$ and stable in temporal terms $(r=, 778)$ with a three week interval. It also converges moderately with the Academic Procrastination Scale Tuckman ( $r=594)$. CONCLUSIONS: The academic procrastination test shows favorable results of validity and reliability in Ecuadorian students.

KEY WORDS: Factorial Analysis, reliability, Academic Procrastination, validity.

\section{CÓMO CITAR / HOW TO CITE}

Moreta-Herrera, R., \& Durán-Rodríguez, T. (2018). Propiedades psicométricas de la escala de procrastinación académica (EPA) en estudiantes de psicología de Ambato, Ecuador. Salud \& Sociedad, 9(3), 236-247. doi: 10.22199/S07187475.2018.0003.00003

1. Psicólogo Clínico. Máster Universitario en Psicología. Profesor investigador de la Escuela de Psicología de la Pontificia Univer sidad Católica del Ecuador.

Personal de formación en la Universidad Autónoma de Madrid. E-mail: rmoreta@pucesa.edu.ec

2. Psicóloga Clínica y personal de formación en investigación de la Escuela de Psicología de la Pontificia Universidad Católica del Ecuador.

E-mail: carolina.t.duran.r@pucesa.edu.ec 


\section{INTRODUCCIÓN}

La procrastinación es un patrón de conductas que se caracteriza por demorar 0 aplazar la consumación de una tarea asignada por otros o por sí mismo (Steel, 2007). Esta surge tras el éxito en realización de una tarea, que por factores ambientales, se la realizó en un tiempo reducido, poco antes del tiempo de entrega y no mostró consecuencias. Así, el procrastinador interactúa en un juego en el que la recompensa, se mezcla con la tensión y la adrenalina que provoca la postergación y la distensión tras la realización de la tarea en el último momento (Alba-Leonel \& HernándezFalconi, 2013). Así, la persona prescinde de la responsabilidad en la toma de decisiones ante la actividad que se requiere finalizar. Este comportamiento puede consistir en un estilo de vida de aplazamiento continuo de tareas y de decisiones que deben tomarse (Moreno, 2007) y que provoca falta de bienestar e incomodidad personal.

En el campo del aprendizaje, este tema cobra interés e importancia para el desarrollo de la psicología y la educación. Es así que, el análisis de esta problemática cuenta con trabajos en países de la región como México (Alba-Leonel \& Hernández-Falconi, 2013); Perú (Álvarez, 2010; Alegre (2014); Domínguez y otros (2014), Argentina (Furlan, Heredia, Piemontesi, \& Tuckman, 2012) y Colombia (Pardo, Perilla \& Salinas, 2014); sin embargo, en el Ecuador, este es limitado, por la ausencia de estudios significativos de la dinámica de la Procrastinación y sus implicaciones con otras variables sociales, escolares o psicológicas y principalmente por la falta de pruebas de medición ajustados a criterios de fiabilidad y validez.

\section{Procrastinación y procrastinación académica}

La procrastinación es un fenómeno contemporáneo, ocasionado por las características sociales actuales, la tecnología, el estilo de vida de los individuos (cargado de compromisos y con fechas de término de actividades, que a la larga inciden en el aumenta de los niveles de postergamiento) (Milgram, 1992); con una relevancia significativa ante la incompatibilidad con la concepción de los sistemas productivos eficientes, en los que la dilación de las actividades toma un tinte negativo (Ferrari, Johnson \& McCown, 1995) y se convierte en una problemática que cuesta tiempo y dinero (Angarita, 2014). Sin embargo, es una práctica habitual (como práctica generalizada o selectiva) que llega a convertirse en un impedimento y de contrariedad para la consecución de los objetivos personales.

Cuando la dilación de actividades se ejecuta en el ambiente escolar exclusivamente, entonces toma el nombre de Procrastinación Académica (PA). Que comprende el aplazamiento continuo de las tareas académicas obligatorias por parte de los estudiantes en cualquier nivel educativo y que se las deja para el último minuto a la entrega (Moreno, 2007). El individuo piensa en realizar la tarea sin embargo conductualmente rehúye del inicio de la misma y frecuentemente justifica que no halla ni el 'escenario' ni el 'tiempo' para su ejecución y culminación; así, se contempla como una conducta de evitación optativa frente a una tarea determinada (Quant \& Sánchez, 2012). El aplazamiento de las tareas funge como un reforzador y un obstáculo para avanzar en el progreso académico e influye en la perdida de motivación para iniciar tareas a futuro (Sánchez, 2010, p. 90). Los educandos posponen los deberes y responsabilidades en sus estudios por no conseguir reforzadores de la conducta contraria. En general entre el $15 \%$ y el $20 \%$ de las personas adultas procrastinan en su entorno académico junto con dificultades en la autoeficacia, tensión y problemas en el confort de la propia persona (González \& Sánchez, 2013). Los estudios actuales muestran incluso que el sexo es invariante con respecto a esta condición (Alegre, 2014; Pardo, Perilla \& Salinas, 2014). 
La PA comprende un patrón conductual y escolar disfuncional, en el que se retarda el inicio de la realización de una tarea (Furlan et al., 2012) y existe la intención expresa de ejecutarla durante periodos de tiempo cortos minutos antes de su entrega para pretender cumplir con la misma (Vallejos, 2015). Al parecer comprende un fenómeno en el que no se han desarrollado estrategias adaptativas.

Así, el estudiante toma y da valor a las acciones que necesita realizar y las analiza en función del tiempo de trabajo a realizar, el tiempo de entrega y la gratificación a obtener. Decantándose por aquellas de gratificación rápida e inmediata y facilidad, que por las representan esfuerzo e inversión de tiempo y energía (e.g. estudiar para rendir las evaluaciones, entrega de tareas de asignaturas complejas, otros) (Dewitte y Lens, 2000; Steel, 2007). En consecuencia el estudiante experimenta preocupación por su acción, rinde menos de lo necesario $\mathrm{n}$ la ejecución de la tarea y tarda en la entrega de las mismas al concluir que no está preparado para dicha labor.

Además, existe una intención de 'excusar' su conducta con coartadas para disminuir el impacto emocional por la inobservancia de su rol estudiantil. También para evitar la culpa cuando se encuentra frente a la tarea y para justificar sus retrasos (Sánchez, 2010) y su dinámica comportamental. Por ejemplo, se intenta por parte del propio estudiante de otorgarse un ilusorio alegato ante la actitud dilatoria, asegurando que se posee un ritmo 'especial' para ejecutar las ocupaciones que le competen. De esta manera la persona tiene la intención de realizar en el momento la tarea que está aplazando, concluyendo que el dilatar es una acción estratégica por buenos motivos (Guzmán, 2013).

\section{Modelos explicativos de la Procrastinación Académica}

La PA es un fenómeno del que existen vertientes que explican su dinámica, así por ejemplo, desde una perspectiva constructivista, esta se da por la relación docente-alumno. La forma en que el alumno adquiere conocimientos se genera y se refuerza a través de los años, con mecanismos mentales aprendidos que direccionan la forma de ejecutar una tarea determinada, el estudiante interpreta el mundo según esta construcción y el profesor desarrolla abordajes metodológicos que acompañen y expliquen en la práctica, el sentido y la intención de enseñanza (Villarruel, 2012). En este aspecto, la planificación educativa que se ejecuta para impartir clase está determinada por las particularidades de los estudiantes, es decir, por las exigencias, competencias y motivación del alumno hacia la instrucción (Pintrich \& Schunk, 2006). Desde este enfoque entonces, la Procrastinación Académica es la consecuencia de las fallas en este proceso de construcción y consolidación de esta relación, no como un aspecto exclusivo del individuo, sino más bien de carácter grupal.

Por otra parte, desde la perspectiva cognitiva y conductual, se explica como un comportamiento que muestra un defecto en el proceso de autorregulación, por consiguiente está implícito la demora en el arranque y la consumación de una tarea; posponiéndola para una próxima fecha (González y Sánchez, 2013). Entretanto, quienes procrastinan muestran una idea irracional de pretender acabar a futuro, la tarea en el menor tiempo posible y en los últimos momentos que poseen. Además, cuando se alcanzan buenos resultados gracias a la dilación y las excusas engañosas, la creencia irracional se refuerza y se mantiene vigente, por lo tanto, la conducta vuelve a repetirse (Ellis \& Knaus, 1977). Debido a esta creencia, el sujeto se configura metas y objetivos altos y poco realizables. Una de las ideas que priman en la dinámica, es la relacionada con el tiempo de disponibilidad por ejemplo. 
Causas de la procrastinación académica

La explicación de las causas de la PA, son variadas y responden a una dinámica de causalidad multifactorial. Algunas investigaciones recogen entre las múltiples causas a las fallas en las capacidades de autorregulación del individuo (Tuckman, 1990), es decir, los estudiantes evitan o dejan para otro momento la acción de la tarea, misma que puede ser controlada y organizada. El miedo al fracaso escolar (González \& Sánchez, 2013), la aversión a las tareas (Natividad, 2014), los problemas en la gestión del tiempo (Chan, 2011) e incluso el perfeccionismo (Knaus, 1997). Así mismo, también sucede por problemas cognitivos por considerar las tareas de poco valor para el individuo, por la obtención de recompensas menores o a largo plazo, por la percepción del grado de dificultad para realizarlas o por los recursos conductuales 0 cognitivos que se demandan para su realización (Angarita, 2014).

La capacidad de elección y la ejecución de tareas en que radica la importancia de la recompensa y el grado de gratificación obtenido (Argumedo et al., 2009). También se explica por los hábitos diarios, los niveles de atención de las personas, la motivación para su ejecución, la capacidad para tolerar la frustración (Milgram, 1992) y la capacidad para controlar o regular sus sentimientos y emociones (Moreta-Herrera, DuránRodríguez \& Villegas-Villacrés, 2018). Mientras que desde el contexto académico, la dilación de las tareas es debido a la pérdida en la valoración de la importancia que tienen las tareas 0 actividades de las que se pueden obtener mayores beneficios y satisfacciones a corto y mediano plazo (Álvarez, 2010).

\section{Efectos en el individuo}

Entre los efectos más destacados de la PA se encuentran el estrés y el rendimiento académico. En el primero la falta de tolerancia a la frustración en los estudiantes está acompañada de la necesidad constante de recompensas inminentes. $\mathrm{El}$ procrastinador pospone las tareas para evitar esta frustración y experimenta ansiedad relacionada con su actitud, quizás busca la propia ansiedad y el alivio que le produce el cumplir con la tarea en el último minuto (AlbaLeonel \& Hernández-Falconi, 2013). Así, el individuo desea completar sus responsabilidades en un periodo corto, en un esfuerzo frenético por ganarle al tiempo y superar al sentimiento de fracaso por no llegar a la meta. Para evitarlo, el procrastinador termina sus actividades probablemente bajo condiciones de elevado estrés (Furlan et al., 2012).

En cuanto al rendimiento académico, la PA conlleva a un bajo desempeño, por los plazos que se establecen en la estrega de las tareas que son relegadas para evitar la actividad. Comprende una estrategia que resulta emocionalmente costosa y de autosabotaje (Difabio, 2011) y puede llevar al estudiante al fracaso y la deserción universitaria. Además, trae problemas en el estudio, la carrera profesional y la vida personal, más aún en el actual entorno social, industrial y moderno en el tiempo está asociado con el valor económico (Furlan, Ferrero \& Gallart, 2014).

A más de ello, como la dilación es una conducta habitual y de gran presencia a nivel universitario puede afectar a la persona en sus relaciones sociales debido a los pensamientos de desamparo e ineficacia, pues no le permiten al estudiante la consecución y el desarrollo de la actividad académica (Pardo, Perilla \& Salinas, 2014) y ocasiona con el tiempo una imagen negativa ante sus pares. También, hay que considerar que la PA afecta en la toma de decisiones, tanto para continuar con los estudios realizados como para satisfacer los requerimientos que su entorno le exija (Chan, 2011, p. 54). En este aspecto, el alivio momentáneo de la ansiedad (al postergar la tarea) revierte a largo plazo en el incremento de dudas sobre la capacidad del procrastinador, además incide en las emociones depresivas y la adhesión a un 
patrón evitativo, pues se empobrece el desempeño ya que se cree erróneamente que se tendrá más habilidad, inspiración y se hará un trabajo de mejor calidad si se deja para el último minuto (Argumedo et al., 2009).

Como consideraciones a esta revisión, es necesario destacar, que la procrastinación no lleva al incumplimiento tácito de una tarea 0 al alcance de la meta explícitamente, sino que se la logra bajo condiciones de elevado estrés en el último momento (Alegre, 2014). Es decir, se señala un acto deliberado e injustificado de aplazar la actividad. Además, quienes procrastinan lo hacen porque sus conductas de dilación se refuerzan por factores del ambiente (Vallejos, 2015). También cabe aclarar, que una tarea que se pospone no es estrictamente una conducta procrastinadora, como por ejemplo el aplazamiento debido a la asignación de tareas o trabajos 'excesivos', que obligan al estudiante a considerar unas tareas en favor de otras para su ejecución; sino que deben cumplir con la dinámica tanto cognitiva, afectiva y conductual para considerarla como tal.

\section{Medición y evaluación de la procrastinación académica}

En el intento por el desarrollo de instrumental de medición de la PA, se presentan diversas propuestas, de las que por sus trabajos realizados en Sudamérica y en la región destacan, el Student Procrastination Scale (Busko, 1998) compuesta por las escalas de General Procrastination (12 ítems) y Academic Procrastination (16 ítems), el que se realizó en 112 estudiantes de grado (33 hombres y 79 mujeres) de la Universidad de Guelph, Canadá en inglés. Se evidenció que la prueba es consistente con $\alpha=, 82$ (escala general) y $\alpha=, 86$ (escala académica). Y que posteriormente se tradujo al castellano y se adaptó al contexto regional.

Destaca entre estos estudios, los de fiabilidad y validez en el Perú realizado de Álvarez (2010) en estudiantes de secundaria en la versión original de 16 ítems traducida al castellano para la PA; en que se encontró una consistencia interna de $\alpha=.8$; con una estructura unifactorial que explica el 23,89\% de la varianza. También, el trabajo de Alegre (2014) en estudiantes universitarios peruanos, con buena consistencia interna de $\alpha=, 78$; y con similar estructura unifactorial que explica el $42,8 \%$. Sin embargo, aunque estos estudios analizan la estructura factorial del EPA, únicamente lo hacen a nivel exploratorio, dejando de lado pruebas confirmatorias que respalden los hallazgos encontrados.

Un estudio más completo es el de Domínguez, Villegas y Centeno (2014), pero con una versión reducida del EPA al encontrarse que no todos los ítems despuntaron el límite mínimo de la homogeneidad. Así, el AFE reveló una estructura bifactorial de la prueba con factores de: Autorregulación Académica (AA) y Postergación de Actividades (PAc) que en conjunto logran explicar el $49,55 \%$ de la varianza y con valores confirmatorios de $X^{2}{ }_{(53)}=176,3 ; p<0,001 ; C F I=1 ; G F I=, 97 ;$ AGFI= ,96; RMR= ,64 y RMSEA= ,78 IC: $90 \%$ [,66 - ,91]. La consistencia interna encontrada resultó ser aceptable con valores de $\alpha=, 816$ (escala global); $\alpha=, 821$ (factor 1) y $\alpha=.752$ (factor 2). Según lo propuesto por los autores, la PA se compone mayoritariamente por un conjunto de difiultades en el proceso de autorregulación del aprendizaje (AA) como un proceso activo para manejar las cogniciones y las motivaciones con respecto a las actividades escolares y por la postergación de actividades (PA), que responde a las conductas de dilación de las actividades como resultante de las dificultades de regulación autónoma.

También se destaca la Escala de Procrastinación Académica de Tuckman (ATP) (Tuckman, 1990), del que existe una adaptación argentina en 277 estudiantes universitarios de Córdoba, Argentina (ATPS) (Furlan et al., 2012) igualmente con 
resultados de fiabilidad aceptables para la población argentina $\alpha=, 87$ en una estructura de carácter unifactorial.

\section{Objetivos e Hipótesis de trabajo}

Como se enunció previamente, es imperante ampliar información y conocimientos sobre la dinámica de la Procrastinación Académica, así como del desarrollo de instrumental adecuado que permita su medición y que en el caso del Ecuador es inexistente, por lo que el objetivo de este estudio, es evidenciar las propiedades psicométricas de la Escala de Procrastinación Académica (EPA) en una muestra de estudiantes universitarios de la ciudad de Ambato, Ecuador para su aplicabilidad generalizada y que cuente con criterios de validez y fiabilidad. De esta manera, se estima que el modelo propuesto por Domínguez, Villegas y Centeno (2014) es el que mejor se ajusta a las puntuaciones obtenidas en estudiantes ecuatorianos.

\section{MÉTODO}

\section{Diseño}

El estudio actual es un diseño instrumental de la estructuración factorial, las propiedades psicométricas de la Escala de Procrastinación Académica (EPA) en el que se incluye el análisis de la consistencia interna de los ítems, la fiabilidad temporal 0 estabilidad de la prueba en dos intervalos de tiempo y la validez de convergencia en un grupo de estudiantes universitarios del Ecuador.

\section{Participantes}

En el estudio participaron 290 estudiantes de la Escuela de Psicología de la Pontificia Universidad Católica del Ecuador - Ambato, que son aproximadamente al $75 \%$ de la población total de la escuelas; con edades que fluctúan entre los 17 a los 30 años (M= 20,8 años; Ds= 1,9). El $23,4 \%$ de la muestra son hombres y el 76,6\% mujeres; el $95,8 \%$ se autodefinen étnicamente como mestizos y el $1,4 \%$ como indígenas, afro-ecuatorianos y blancos respectivamente. Además, un 52,1\% de los participantes se encuentran domiciliados en la ciudad de Ambato mientras el otro $47,9 \%$ viajan diariamente de otras ciudades de la zona.

El $59,3 \%$ de los participantes 10 conforman estudiantes de la carrera de Psicología Clínica y el $40,7 \%$ los estudiantes de la carrera de Psicología Organizacional. Asimismo los participantes cursan sus estudios entre el primer nivel $(13,1 \%)$ y el décimo nivel (4.8\%) de formación profesional, encontrándose la mayor participación en el estudio los estudiantes del segundo nivel $(17,9 \%)$. Un $53,4 \%$ de los estudiantes poseen subsidio de estudios (becas de excelencia o ayudas económicas) para cubrir los gastos de sus estudios. Los antecedentes de migración universitaria de otras carreras y universidades son del $34,4 \%$. La tasa de repetición de nivel se encuentra en el $5,5 \%$.

\section{Instrumentos}

Escala de Procrastinación Académica (EPA). Instrumento diseñado por Busko (1998), en la versión adaptada al castellano de Álvarez (2010) y reducida de 12 ítems (Domínguez, Villegas \& Centeno, 2014). La misma que se responde es una escala Likert de 5 opciones. El uso de esta prueba y en la versión reducida se justifica por el uso de estimaciones exploratorias y confirmatorias previas de la estructura factorial junto con las características de la muestra en las que se analizó.

Escala de Procrastinación Académica de Tuckman, versión argentina (ATPS). Esta comprende un instrumento que en su forma original en inglés constó inicialmente de 72 ítems, que para tras varios análisis se lo redujo a 16 ítems (Tuckman, 1990). Se utilizó la Adaptación Argentina de la Escala de Tuckman (ATPS) (Furlan et al., 2012) en su versión de 15 ítems. El AFC muestra una estructura factorial unidimensional con una consistencia interna alta $(\alpha=, 87)$. El uso de esta escala en el estudio actual tiene como 
finalidad realizar el análisis de validez de convergencia con el EPA.

\section{Procedimiento}

La evaluación se lo realizó de manera grupal, previamente se socializó acerca de los objetivos del proyecto en los salones de clase de la Escuela de Psicología y se les invitó a participar de manera voluntaria a los estudiantes. Quienes decidieron participar leyeron y firmaron una carta de aceptación para la participación en el estudio para luego proceder a la realización de la prueba. Posteriormente, se procedió a la depuración de la información para la elaboración de una base de datos, la gestión informática de los datos y la comprobación estadística de las hipótesis de trabajo.

\section{Análisis de resultados}

En la evaluación de la estructura del EPA se utilizó el Análisis Factorial Exploratorio (AFE) y Confirmatorio (AFC). En el primero se consideró las pruebas de adecuación muestral de Kaiser-Meyer-Olkyn (KMO; Kaiser, 1974) y de aplicabilidad para el análisis factorial con la Prueba de Bartlett $\left(X^{2}\right)$ (Bartlett, 1950). También se incluye un análisis de las comunalidades $\left(h^{2}\right)$. Para finalmente realizar la extracción de los factores con el Método de Mínimos Cuadrados no Ponderados (MCNP), pues se parte del supuesto de no normalidad multivariante (Lorenzo-Seva, 1999) y la rotación con el método Varimax.

En el segundo análisis confirmatorio, se utilizó el Método de Máxima Verosimilitud del que se analizó el ajuste absoluto con el índice corregido (S-B $X^{2}$ ) de Satorra-Bentler (2001) y la razón Chi Cuadrado normado (S$\left.B X^{2} / d f\right)$, en el que se espera obtener valores inferiores a 4 para considerar un ajuste aceptable (Hu \& Bentler, 1999). Además se usó los Índices de Bondad de ajuste (GFI) y Ajustado de bondad de ajuste (AGFI) en el que valores de un buen ajuste se encuentran a partir de ,8 (Hair, Anderson, Tatham \& Black, 2004). También se usó el Índice de
Ajuste Comparativo (CFI) en el que valores ,9 o más son aceptables (Byrne, 2006) y el Error Cuadrático medio de Aproximación (RMSEA) en el que se considera ajustes aceptables con puntaciones entre ,06 y, 08 (Byrne, 2006).

Por otra parte, los cálculos de fiabilidad se realizaron por medio de la consistencia interna de los ítems con el Coeficiente de Cronbach (a) y el coeficiente Omega ( $\omega$ ) en el que se consideran puntuaciones óptimas los valores iguales o superiores a .8. La estabilidad temporal de la prueba en dos tomas en 48 participantes con la técnica Test-Retest. Y por último la convergencia del EPA con la prueba ATPS (Furlan et al., 2012), pues miden procrastinación estudiantil y en el que se utilizó un análisis de correlación (r).

La gestión informática de los datos se realizó con el empleo del software FACTOR 9.2 (Lorenzo-Seva \& Ferrando, 2013) para el AFE y el $R$ Comander en la versión 2.23 ( $R$ Core Team, 2015) para el AFC y las pruebas de fiabilidad.

\section{RESULTADOS}

\section{Análisis Factorial}

Análisis exploratorio. Los análisis de adecuación muestral de los ítems (KMO y Bartlett) que se refleja en la tabla 1, muestran que los puntajes de los ítems se encuentran dentro de los rangos aceptables para la conformación de factores. En cuanto a las comunalidades entre sí de los ítems, estos fluctuaron entre $h^{2}=0,821$ en el ítem 6 y $h^{2}=$ ,186 en el ítem 2 señalando así la ausencia de multicolinealidad. Es probable que se requiera la eliminación de ítems (2) para mejorar la factorización de la prueba tras el análisis confirmatorio.

Ya en el análisis exploratorio propiamente, la extracción a través del MCNP y con la rotación Varimax, muestran que la prueba con dos factores logra explica la varianza en un $53,1 \%$. En el análisis entre 
los factores, la Autorregulación del Aprendizaje explica el $39,6 \%$ de la varianza con ocho ítems; con cargas factoriales que fluctuaron entre, 707 y, 321 ; mientras que en el factor de Postergación de Actividades, explica el $13,5 \%$ de la varianza con tres ítems, con cargas que fluctuaron entre, $903 \mathrm{y}$ , 552 .

TABLA 1.

Análisis factorial exploratorio del EPA con el Método de Mínimos Cuadrados no Ponderados y Rotación Varimax.

\begin{tabular}{lccc}
\hline \multirow{2}{*}{ İtems } & \multirow{2}{*}{$\mathrm{h}^{2}$} & \multicolumn{2}{c}{ Componente } \\
\cline { 2 - 4 } & & $\mathrm{AA}$ & $\mathrm{PCA}$ \\
\hline İtem 11 &, 577 &, 707 &, 279 \\
İtem 05 &, 603 &, 702 &, 332 \\
İtem 12 &, 454 &, 667 &, 095 \\
İtem 08 &, 404 &, 633 &, 051 \\
İtem 10 &, 409 &, 613 &, 181 \\
Ítem 03 &, 294 &, 537 &, 074 \\
İtem 09 &, 382 &, 530 &, 319 \\
İtem 04 &, 201 &, 435 &, 106 \\
İtem 02 &, 186 &, 321 &, 288 \\
İtem 06 &, 821 &, 080 &, 903 \\
İtem 07 &, 675 &, 122 &, 813 \\
İtem 01 &, 494 &, 435 &, 552 \\
\hline Varianza & - &, 396 &, 135 \\
\hline KMO: ,841 & \multicolumn{3}{|}{} \\
Bartlett: 1005; Df: 45; p< 0,001 &
\end{tabular}

Análisis Confirmatorio. Se probaron tres modelos factoriales, para considerar cuál de ellos presenta un mejor ajuste en los estudiantes ecuatorianos. El primer modelo de tipo unifactorial, con los doce ítems, el segundo con dos factores correlaciones; propuestos por Domínguez y otros (2014) en estudiantes peruanos; y un tercer modelo factorial de dos factores correlacionados con 11 ítems (2do eliminado debido a una baja comunalidad).
Los resultados del AFC como se mira en la tabla 2, con el método de Máxima Verosimilitud muestran que el segundo modelo factorial, es el que presenta un mejor ajuste de la prueba a la muestra de estudiantes ecuatorianos con criterios de ajuste robusto aceptables, así como en los distintos índices de ajuste.

TABLA 2.

Modelos factoriales confirmatorios del EPA con el Método de Máxima Verosimilitud.

\begin{tabular}{lccccccc}
\hline Modelo & B-S X & Df & B-S X2/df & CFI & GFI & AGFI & RMSEA \\
\hline Unifactorial & $232,6^{* * *}$ & 54 & 4,3 &, 73 &, 84 &, 77 &, $120[, 13-, 11]$ \\
Dos factores (12 ítems) & $119,1^{* * *}$ & 53 & 2,3 &, 90 &, 92 &, 89 &, $076[, 06-, 09]$ \\
Dos factores (11 ítems) & $119,2^{* * *}$ & 43 & 2,8 &, 91 &, 93 &, 89 &, $083[, 07-, 10]$ \\
\hline
\end{tabular}

Nota: 290 observaciones

${ }^{* * *} p<, 001$

Análisis de consistencia interna y estabilidad temporal

En la tabla 3, el cálculo de la fiabilidad muestra que el factor de Autorregulación Académica con nueve ítems tiene una consistencia elevada; mientras que en la Postergación de Actividades esta es moderada alta. A nivel global del EPA, la fiabilidad resultante es alta. Al existir un mecanismo de doble evaluación de la fiabilidad con la prueba regular (a) y la 
alternativa ( $\omega)$, se concluye que las consistencias alcanzadas son adecuadas y permiten disipar dudas planteadas sobre la consistencia en población ecuatoriana.

TABLA 3.

Análisis de consistencia interna del EPA.

\begin{tabular}{lccc}
\hline Factores & İtems & Alfa & $\omega$ \\
\hline Autorregulación académica & 9 &, 80 &, 80 \\
Postergación de actividades & 3 &, 77 &, 79 \\
\hline Global EPA & 12 &, 83 & 83 \\
\hline Nota: 290 observaciones. & & &
\end{tabular}

Por otra parte, acerca de la estabilidad temporal de la prueba considerando un lapso de tres semanas desde la primera toma en los participantes para conocer la presencia 0 no de cambios relevantes o significativos. La tabla 4, muestra que ni en los factores del EPA, así como en el global de la misma, se encontraron diferencias estadísticamente significativas $(p<, 05)$ entre las dos tomas de la prueba. Es decir, no se aprecian variaciones temporales significativas. A la luz de los datos analizados del EPA, también se concluye que es válida en términos de estabilidad temporal.

\section{TABLA 4.}

Validez de la fiabilidad como estabilidad de la medida en intervalos en tiempo del EPA.

\begin{tabular}{lccccccc}
\hline \multirow{2}{*}{ Factores } & \multicolumn{2}{c}{ Test } & \multicolumn{2}{c}{ Re-test } & \multirow{2}{*}{$\mathrm{i}$} & \multirow{2}{*}{$($ (47) } \\
\cline { 2 - 5 } & Media & Desv. & Media & Desv. & &, $484^{* * *}$ &,- 816 \\
Autorregulación académica & 19,2 & 5,0 & 19,7 & 5,2 & \\
Postergación de actividades & 8,4 & 3,0 & 8,2 & 3,0 &, $794^{* * *}$ &, 599 \\
\hline Global EPA & 27,6 & 7,0 & 27,9 & 7,8 &, $778^{* * *}$ &,- 435 \\
\hline Nota: ${ }^{*} p<.05 ;{ }^{* *} p<.01 ;{ }^{* *} p<.001$ & & & & & & &
\end{tabular}

\section{Validez de convergencia del EPA}

Como se puede observar en la tabla 5 , la comprobación de la validez de convergencia del EPA se la realiza con la prueba ATPS, pues las mismas se encargan de medir la Procrastinación estudiantil. En el análisis se encontró que el ATPS converge a través de correlaciones moderadas positivas y significativas con los factores de Autorregulación Académica y de Postergación de Actividades. Y asimismo, lo hace también con la puntuación global. Es decir, que la prueba goza de una adecuada validez con otra prueba similar en estudiantes ecuatorianos.

TABLA 5.

Validez de convergencia del EPA con relación al ATPS.

\begin{tabular}{cccc}
\hline & Autorregulación & Postergación & EPA \\
\hline ATPS &, $461^{* *}$ &, $618^{* *}$ &, $594^{* *}$ \\
\hline Nota: ${ }^{* *} p<.01$ & & &
\end{tabular}

\section{DISCUSIÓN}

El objetivo del presente reporte es confirmar el modelo factorial de dos componentes de la Escala de Procrastinación Académica de
Busko (1998) adaptado por Domínguez, Villegas y Centeno (2014) para la población ecuatoriana; a más de las propiedades psicométricas de fiabilidad y la validez de convergencia. La estructuración factorial del 
EPA muestra una conformación de dos factores correlacionados que explican entre sí un $51,3 \%$ de la varianza y que corroboran lo propuesto por Domínguez y otros (2014) con dos factores correlacionados, junto con la revisión de los criterios de ajuste robusto y los índices de ajuste sugeridos por $\mathrm{Hu}$ y Bentler (1999); Hair, Anderson, Tatham y Black (2004) y Byrne (2006). Estos resultados permiten confirmar la hipótesis planteada.

En cuando a la consistencia interna del EPA, los resultados muestran que la prueba de forma global es consistente en la población ecuatoriana. Hallazgos similares en otros contextos, se encuentran en el estudio original de Busko (1998) aplicado en canadienses; de Álvarez (2010) y de Alegre (2014) en universitarios peruanos y Pardo, Perilla y Salinas (2014) en colombianos. A más de ello, en los factores constitutivos del EPA, la Autorregulación Académica y la Postergación de Actividades también muestran consistencia, como previamente Domínguez y otros (2014) lo encontraron. En este aspecto, se infiere que la prueba es fiable y consistente para la población ecuatoriana y congruente en similar situación con otras poblaciones referenciales.

En cuanto a la validez, a nivel de estabilidad en dos intervalos de tiempo con tres semanas de diferencia, se encontró que la prueba es estable, asumiendo la ausencia de cambios significativos entre los participantes para la segunda aplicación. Mientras que en la convergencia, el EPA es convergente con pruebas similares medidas en estudiantes como lo es el ATPS (Furlan et al., 2012). En conclusión, los hallazgos de validez muestran que la prueba es válida en términos de estabilidad y de convergencia. Sin embargo, cabe la pena señalar que no se encuentran datos referenciales previos con los que se puedan contrastar estos resultados, por lo que esto representa un avance significativo para la medición confiable de la procrastinación académica.
Entre las implicaciones de los hallazgos, se pueden considerar que el EPA comprende una herramienta válida con una estructura bifactorial consistente para la evaluación del PA en estudiantes universitarios que goza de fiabilidad y convergencia. Cabe además señalar la importancia que tiene la autorregulación del aprendizaje en la explicación de la procrastinación académica, que a través del estudio se corrobora con lo expuesto por Tuckman (1990); González y Sánchez (2013); y Moreta-Herrera, DuránRodríguez \& Villegas-Villacrés (2018) al considerarlos más como un problema de adaptabilidad que de motivación 0 de ausencia de recursos cognitivos y que en este aspecto la postergación es más bien una respuesta reactiva a los procesos de desrregulación del contexto académico; lo que refuerza el aspecto constructivista de la procrastinación como fue señalado por Villarruel (2012).

Se debe considerar que las discusiones factoriales de Alegre (2014) y Álvarez (2010) no aplicaron análisis confirmatorios para corroborar la unidimensionalidad de la misma, por lo que existe la duda planteada acerca de la estructuración del constructo. $Y$ los resultados de este trabajo más bien refuerzan la tendencia a comprender el fenómeno de la procrastinación de manera bifactorial propuesto por Domínguez, Villegas y Centeno (2014), pues los ensayos en distintos segmentos poblacionales de estudiantes de una misma región (Perú y Ecuador) dan evidencia de dicha condición. A más, como un elemento a considerar, al parecer estamos tratando con una variable con características transculturales que necesita ser tratados a futuro.

Por último, al considerarse que la prueba es aplicable a estudiantes ecuatorianos, esto permite el desarrollo a futuro de estudios de la PA asociadas a aspectos internos como la motivación, la afectividad, la autoeficacia; la ansiedad 0 el estrés relacionadas con el entorno académico; los rasgos de personalidad; o la regulación académica, la 
gestión del tiempo, la innovación educativa, el rendimiento académico y la mejora educativa universitaria para los estudiantes.

\section{Limitaciones y líneas de investigación a futuro}

Entre las principales limitaciones del presente estudio podemos considerar en primero a las características de la muestra, pues se trabajó exclusivamente con estudiantes de psicología de una universidad cofinanciada de la ciudad de Ambato. Por lo que a futuro se requiere confirmar estos hallazgos con estudiantes de otras carreras y centros de estudio, así como en adolescentes escolarizados, de educación técnica o de formación continua, otros. Sobre todo para obtener una propiedad inferencial de la prueba de forma generalizada.

En segundo lugar, en el análisis de la estructura factorial de la prueba, tanto el AFE como el AFC no se realizaron como estudios independientes segmentando a la muestra, aunque no es un requisito indispensable, es recomendable que a futuro se tracen estudios confirmatorios considerando este aspecto. Finalmente, no se contemplaron otros análisis de validez de la prueba, como por ejemplo la discriminante, para determinar si la prueba tiene o no la capacidad de segmentar a la población normal de la vulnerable que pueda presentar problemas escolares relacionados al aprendizaje o la adaptación socio-escolar.

\section{REFERENCIAS}

Alba-Leonel, A., \& Hernández-Falconi, J. (2013). Procrastinación académica en estudiantes de la Escuela Nacional de Enfermería y Obstetricia, UNAM. Memorias TECNyMED, 1-9.

Alegre, A. (2014). Autoeficacia y procrastinación académica en estudiantes universitarios de Lima Metropolitana. Educación y Psicología de la USIL, 1(2), 57-82.

Álvarez, O. (2010). Procrastinación general y académica en una muestra de estudiantes de secundaria de Lima metropolitana. Persona, 13, 159-177.
Angarita, L. (2014). Algunas relaciones entre la procrastinación y los procesos básicos de motivación y memoria. Revista Iberoamericana de Psicología: ciencia y tecnología, 7(1), 91-101.

Argumedo, D., Díaz, K., Calderón, A., \& Díaz, J. (2009). Procrastinación: Una aproximación desde los estilos de personalidad. En D. Herrera (Ed.), Teorías contemporáneas de la motivación. Una perspectiva aplicada (págs. 193-212).

Bartlett, M. S. (1950). Tests of significance in factor analysis. British Journal of Statistical Psychology, 3(2), 77-85. Doi: 10.1111/j.2044-8317.1950.tb00285.x.

Batista-Fogueta, J., Coenders, G., \& Alonso, J. (2004). Análisis factorial confirmatorio. Su utilidad en la validación de cuestionarios relacionados con la salud. Medicina Clínica, 122(Supl 1), 21-27.

Busko, D. A. (1998). Causes and consequences of perfectionism and procrastination: $A$ structural equation model. Tesis de maestría no publicada. Guelph, Ontario: University of Guelph.

Byrne, B. M. (2006). Structural equation modeling with EQS. Basic concepts, applications, and programming (2nd ed.). Hills Dale, $\mathrm{NJ}$ : Lawrence Erlbaum Associates.

Chan, L. (2011). Procrastinación académica como predictor en el rendimiento académico en jóvenes de educación superior. Temática Psicológica, 7(1), 53-62.

Difabio, H. (2011). Las funciones del tutor de la tesis doctoral en educación. Revista Mexicana de Investigación Educativa, 16(50), 935-959.

Domínguez, S., Villegas, G., \& Centeno, S. (2014). Procrastinación académica: Validación de una escala en una muestra de estudiantes de una universidad privada. Liberabit, 20(2), 293-304.

Ellis, A., \& Knaus, W. (1977). Overcoming procrastination. Nueva York: Signet Books.

Ferrando, P. J., \& Anguiano-Carrasco, C. (2010). El análisis factorial como técnica de investigación en psicología. Papeles del Psicólogo, 31(1), 18-33.

Ferrari, J., Johnson, J., \& McCown, W. (1995). Procrastination and task avoidance: theory, research, and treatment. New York: Plenum Press.

Furlan, L., Ferrero, M., \& Gallart, G. (2014). Ansiedad ante los exámenes, procrastinación y síntomas mentales en 
estudiantes universitarios. Revista Argentina de Ciencias del Comportamiento, 6(3), 31-39.

Furlan, L., Heredia, D., Piemontesi, S., \& Tuckman, B. (2012). Análisis factorial confirmatorio de la adaptación argentina de la escala de procrastinación de Tuckman (ATPS). Perspectivas en Psicología(9), 142-149.

González, M., \& Sánchez, A. (2013). ¿Puede amortiguar el engagement los efectos nocivos de la procrastinación académica? Acción Psicológica, 10(1), 117-134.

Guzmán, D. (2013). Procrastinación una mirada clínica (Tesis de maestría). Barcelona: Instituto Superior de Estudios Psicológicos.

Hair, J. F., Anderson, R. E., Tatham, R. L., \& Black, W. C. (2004). Análisis multivariante. Madrid, España: Prentice Hall.

Hu, L., \& Bentler, P. M. (1999). Cutoff criteria for fit indexes in covariance structure analysis: Conventional criteria versus new alternatives. Structural Equation Modeling, 6(1), 1-55.

Kaiser, H. F. (1974). An index of factorial simplicity. Psychometrika, 39(1), 31-36. http://dx. doi.org/10.1007/BF02291575.

Knaus, W. (1997). Superar el hábito de posponer. RET, Revista de Toxicomanías, 13, 13-16.

Lorenzo-Seva, U. (1999). Promin: a method for oblique factor rotation. Multivariate Behavioral Research, 34, 347-356.

Lorenzo-Seva, U., \& Ferrando, P. (2013). FACTOR 9.2 A Comprehensive Program for Fitting Exploratory and Semiconfirmatory Factor Analysis and IRT Models. Applied Psychological Measurement, 37(6), 497498.

Milgram, N. (1992). El retraso: Una enfermedad de los tiempos modernos. Boletín de Psicología, 35.

Moreno, B. (2007). Psicología de la personalidad: procesos. Madrid, España: Thomson.

Moreta-Herrera, R., Durán-Rodríguez, T., \& Villegas-Villacrés, N. (2018). Regulación Emocional y Rendimiento como predictores de la Procrastinación Académica en estudiantes universitarios. Revista de Psicología y Educación, 13(2), 155-166. Doi: 10.23923/rpye2018.01.166.

Natividad, L. (2014). Análisis de la procrastinación en estudiantes universitarios (Tesis doctoral). Valencia: Universidad de Valencia.
Pardo, D., Perilla, L., \& Salinas, C. (2014). Relación entre procrastinación académica y ansiedad-rasgo en estudiantes de psicología. Cuadernos Hispanoamericanos de Psicología, 14(1), 31-44.

Pintrich, P., \& Schunk, D. (2006). Motivación en contextos educativos. Teoría, investigación y aplicaciones (2da ed.). Madrid: Pearson Educación.

Quant, D., \& Sánchez, A. (2012). Procrastinación, procrastinación académica: concepto e implicaciones. Vanguardia psicología, 3(1), 45-59.

$\mathrm{R}$ Core Team. (2015). R: A language and environment for statistical computing. Vienna, Austria: R Foundation for Statistical Computing.

Sánchez, A. (2010). Procrastinación académica: Un problema en la vida universitaria. Studiositas, 5(2), 87-94.

Satorra, A., \& Bentler, P. M. (2001). A scaled difference chi-square test statistic for moment structure analysis. Psychometrika, 66(4), 507-514.

Steel, P. (2007). The nature of procrastination: A meta-analytic and theoretical review of quintessential selfregulatory failure. Psychological Bulletin, 133(1), 65-94.

Tuckman, B. (1990). Group versus goal-setting effects on the self-regulated performance of students differing in selfefficacy. Journal of Experimental Education, 58, 291-298.

Vallejos, S. (2015). Procrastinación académica y ansiedad frente a las evaluaciones en estudiantes universitarios (Tesis). Lima: Pontificia Universidad Católica del Perú.

Villarruel, M. (2012). El constructivismo y su papel en la innovación educativa. Revista de educación y desarrollo, 20, 19-28.

Todos los trabajos publicados en Revista Salud \& Sociedad (ISSNe:0718-7475) están sujetos a una licencia Creative Commons Reconocimiento 4.0 Internacional

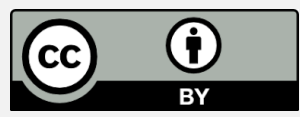

